\title{
homeless is required for RNA localization in Drosophila oogenesis and encodes a new member of the DE-H family of RNA-dependent ATPases
}

\author{
Doreen E. Gillespie and Celeste A. Berg ${ }^{1}$ \\ University of Washington, Department of Genetics, Seattle, Washington 98195-7360
}

\begin{abstract}
The homeless (hls) gene of Drosophila is required for anteroposterior and dorsoventral axis formation during oogenesis. At a low frequency, females homozygous for mutations in hls generate early egg chambers in which the oocyte is positioned incorrectly within the cyst. At a high frequency, late-stage egg chambers exhibit a ventralized chorion. Sequence analysis of the hls cDNA predicts a protein with amino-terminal homology to members of the DE-H family of RNA-dependent ATPases and putative helicases. Similarity of $51 \%$ in the amino-terminal third of the protein was found to two yeast splicing factors, PRP2 and PRP16, and to Drosophila Maleless, which is required for dosage compensation. To analyze Hls function, RNA localization patterns were determined for seven different transcripts in hls mutant ovaries. Previtellogenic transport to the oocyte was unaffected for all transcripts examined. Transport and localization of bicoid and oskar messages during vitellogenic stages were strongly disrupted, and the distribution and/or quantity of gurken, orb, and fs(1)K10 mRNAs were also affected, but to a lesser degree. In contrast, hu-li tai shao and Bicaudal-D transcripts were transported and localized normally in hls mutants. In addition, Kinesin heavy chain: $\beta$-Galactosidase fusion protein failed to localize correctly to the posterior of the oocyte in vitellogenic egg chambers. Examination of the microtubule structure with anti- $\alpha$-Tubulin antibodies revealed aberrant microtubule organizing center movement and an abnormally dense cytoplasmic microtubule meshwork. We discuss potential roles for Hls in organizing a cytoskeletal framework essential for localizing specific RNAs.
\end{abstract}

[Key Words: Localized RNA; RNA-binding protein; anterior/posterior axis; dorsal/ventral axis; microtubule; germ line]

Received April 25, 1995; revised version accepted August 29, 1995.

The rapid development of the Drosophila embryo is facilitated by the establishment of the anteroposterior $(\mathrm{a} / \mathrm{p})$ and dorsoventral $(\mathrm{d} / \mathrm{v})$ axes during oogenesis. The Drosophila egg is formed from an egg chamber composed of 16 interconnected germ-line cells surrounded by somatic follicle cells (for review, see Spradling 1993). By stage 1 ( $\mathrm{S} 1$ ) of oogenesis, the oocyte has been determined and has moved from a central location to the posterior of the 16-cell cyst. The egalitarian, Bicaudal-D (Bic-D), and hu-li tai shao (hts) genes are required to specify the oocyte within the cyst (Steward and Nüsslein-Volhard 1986; Schüpbach and Wieschaus 1991; Yue and Spradling 1992, Ran et al. 1994), whereas dicephalic (dic), spindle- $C$, and armadillo function in positioning the oocyte at the posterior of the egg chamber (Lohs-Schardin 1982; Peifer et al. 1993; González-Reyes and St. Johnston 1994). Cytoskeletal architecture within the egg chamber is important for oocyte determination and po-

${ }^{1}$ Corresponding author. sitioning, as well as for the localization of mRNAs and proteins involved in $\mathrm{a} / \mathrm{p}$ and $\mathrm{d} / \mathrm{v}$ axis formation (Koch and Spitzer 1983; Pokrywka and Stephenson 1991, 1995; Theurkauf et al. 1993; Lin et al. 1994; González-Reyes et al. 1995; Roth et al. 1995; for review, see Theurkauf 1994a).

The 15 nurse cells at the anterior of the developing egg chamber produce RNAs and proteins necessary for the formation and maintenance of the $a / p$ and $d / v$ axes within the oocyte, as well as proteins and organelles that provide essential cellular and developmental functions during embryogenesis. Subcellular localization of the axis-determining RNAs and proteins is important for proper development. This localization requires efficient transport through the nurse cells and oocyte and subsequent anchoring or stabilization within the oocyte. For example, transcripts for the anterior embryonic determinant Bicoid (Bcd) require Exuperantia (Exu), Swallow (Swa), and Staufen (Stau) proteins for transport to, and localization within, the oocyte (for review, see St. Johnston et al. 1989; Stephenson and Pokrywka 1992). 
Transcripts of the posterior determinant Nanos are localized through the activities of the posterior group genes whose products make up the polar granules found at the posterior of the embryo (Mahowald 1962; Wang and Lehmann 1991; for review, see St. Johnston 1993). The first step is the colocalization of Stau protein and oskar (osk) mRNA to the posterior of the oocyte at S8. Localization of each additional component occurs in a stepwise fashion and requires the activity of the earlier genes (Lehmann and Nüsslein-Volhard 1991; for review, see St. Johnston 1993). Both processes, localization of $b c d$ mRNA to the anterior of the oocyte and localization of osk mRNA and Stau protein to the posterior of the oocyte, depend on an organized microtubule network whose orientation is defined by a reciprocal cell-signaling process between germ cells and follicle cells (Pokrywka and Stephenson 1991; Clark et al. 1994; González-Reyes et al. 1995; Roth et al. 1995). This signaling cascade requires the activity of Gurken $(\mathrm{Grk})$, a transforming growth factor- $\alpha$ (TGF- $\alpha$ )-like molecule, and Cornichon (Cni), whose precise molecular function is not known, to act in the germ line and signal to the polar follicle cells (González-Reyes et al. 1995; Roth et al. 1995). Reception of the signal via the epidermal growth factor (EGF)-receptor homolog Torpedo (Top) establishes their fate as posterior. Subsequent signaling from the posterior follicle cells back to the oocyte in a Protein kinase A (PKA)-mediated fashion directs the organization of the microtubule network and leads to the proper localization of embryonic determinants (Ruohola et al. 1991; Lane and Kalderon 1994).

The establishment of the dorsoventral axis also involves localized RNAs and proteins. The first visual manifestation of dorsoventral polarity within the oocyte is the movement of the oocyte nucleus toward the dorsal side of the egg chamber, also via a microtubule-dependent mechanism (Koch and Spitzer 1983; GonzálezReyes et al. 1995; Roth et al. 1995). Subsequently, grk transcripts are localized tightly to the anterodorsal cortex of the oocyte, overlying the oocyte nucleus, in a process dependent on the function of the cappucino, spire, $f_{S}(1) K 10(K 10)$, squid, and orb genes (Neuman-Silberberg and Schüpbach 1993; Christerson and McKearin 1994). Reception of the Grk signal via the EGF receptor in the dorsal follicle cells is required for the synthesis of dorsal eggshell structures and the establishment of dorsoventral polarity in the embryo (Chasan and Anderson 1993; for review, see Schüpbach and Roth 1994). Thus, the $g r k$ pathway acts in establishing both $a / p$ and $d / v$ polarity (for review, see Anderson 1995).

Characterization of RNA localization mechanisms that function in other developmental processes has revealed a selective dependence on cytoskeletal structures. Two basic mechanisms have been described: active transport and anchoring of a message or uniform distribution followed by specific degradation. In a developing Drosophila egg chamber, for example, $\mathbf{a} / \mathbf{p}$ polarity is inherently provided by the relationship of the nurse cells to the oocyte. An transcript entering the oocyte from the nurse cells must enter at the anterior end and, thus, has an efficient staging platform for either retention in the anterior region or specific transport to the posterior pole. In this way, osk and $b c d$ mRNAs are distinguished and differentially localized. Some transcripts, however, are localized in an indirect manner, for example, $H s p 83$ RNA. Transcripts of $H s p 83$ are transported from the nurse cells to the oocyte and are diffuse throughout the oocyte until fertilization. At that time, posterior $H s p 83$ message is protected and the remainder is degraded (Ding et al. 1993a). No role for microtubule scaffolding in Hsp83 message localization has been demonstrated. Similarly, in a Xenopus oocyte, which develops without nurse cells, microtubule organization is critical for the localization of some mRNAs but not others. Diffuse messages of $V g 1$ are localized to the vegetal pole through a microtubule-dependent process (Yisraeli et al. 1990). Subsequent anchoring requires intermediate filaments-cytoskeletal components not found in Drosophila. In contrast, transport of XIsirts, Xcat2, and $X w n t 11$ mRNAs, also localized to the vegetal pole during oogenesis, is resistant to both nocodazole and cytochalasin $B$, suggesting that neither actin nor microtubules are components of the transport mechanisms for these transcripts (Kloc and Etkin 1995). Microtubules also have been implicated in RNA localization in fully differentiated mammalian neuronal cells. tau message, for example, is localized to the cell body and proximal axon region and is associated with the microtubule fraction of extracted cells; tubulin transcripts, restricted to the cell body, do not associate with microtubules (Litman et al. 1994).

The machinery required for RNA localization must provide specificity in transport, localization, and stabilization or degradation, as necessary. In this paper we describe the homeless ( $h l s$ ) gene, whose product is necessary for correct RNA localization during Drosophila oogenesis.

\section{Results}

Isolation of hls mutants

In a large scale $\mathrm{P}\left(\right.$ lacZ,ry $\left.^{+}\right)$screen (described in Karpen and Spradling 1992), we obtained a female sterile line, 3987, in which the P element mapped by in situ hybridization to 89A5,6. Females homozygous for the insertion laid few eggs, and these appeared to be unfertilized. Upon dissection of the ovaries from these females, we observed defects at two stages of oogenesis. At a low frequency, vitellogenic egg chambers were produced in which the oocyte was not located at the usual posterior position in the egg (Fig. 1). In addition, 95\% of late-stage egg chambers were ventralized, at least partially (Fig. 2). We also observed a small number of degenerating egg chambers at all stages (Table 1). We have named the gene hls based partly on the oocyte mislocalization phenotype and partly on the RNA localization phenotype described in subsequent sections of this paper. Females transheterozygous for $h l s^{3987}$ and any of a number of deficiencies uncovering the region (see Materials and methods) pro- 


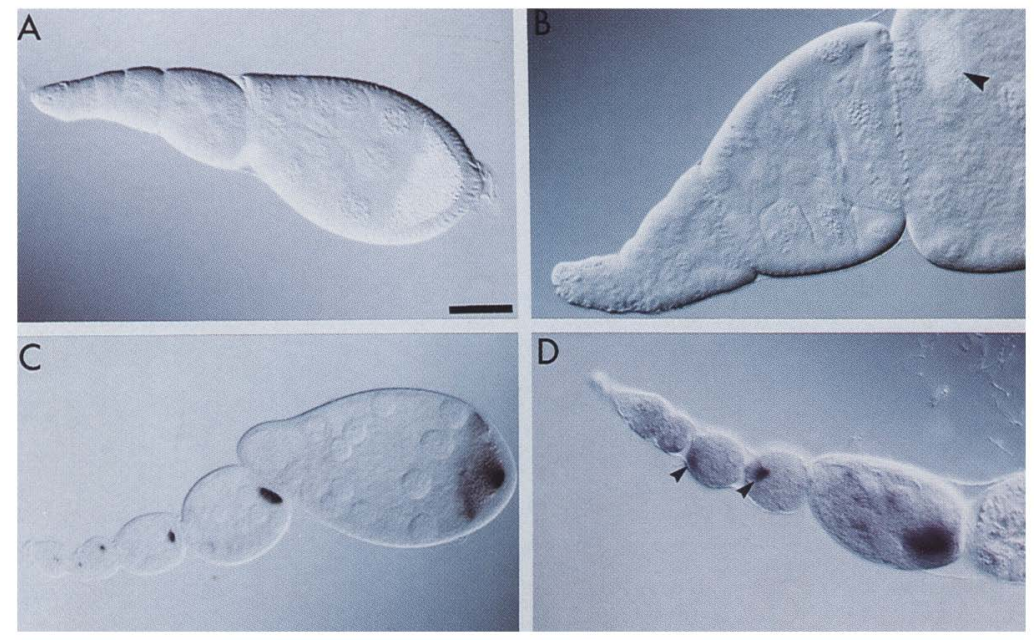

Figure 1. Egg chambers from hls females exhibit mispositioned oocytes. (A) Wild-type ovariole. The S9 egg chamber contains an oocyte located at the posterior. (B) hls ${ }^{3987}$ ovariole. The S8 egg chamber contains a vitellogenic cell located at the anterior (arrowhead). $(C, D)$ Ovarioles probed with osk cDNA sequences to identify previtellogenic oocytes $(C)$ Wild type; $(D) h l s^{3987}$. Arrowheads indicate two mispositioned oocytes. Bar in $A, 20$ $\mu \mathrm{m}$. Note: We distinguish the mispositioned oocyte phenotype of hls egg chambers from that of dicephalic (dic) egg chambers. The term dicephalic implies the presence of nurse cells at each end of the developing egg chamber. In hls egg chambers in which the oocyte is not posteriorly positioned, the oocytes are fully anteriorly localized as frequently as they are centrally located. In addition, some dic eggs generate two micropyles. We have not observed bipolar chorion structures on eggs produced by hls females.

duced fewer late-stage egg chambers because of a higher number of eggs that degenerated at all stages (Table 1). These results suggested that the insertion line was a hypomorph. Two ethylmethane sulfonate (EMS)-induced alleles of $h l s, h l s^{E 616}$ and $h l s^{E 653}$, were kindly provided by Daniel St. Johnston (Wellcome/CRC Institute, Cambridge, UK). When placed in trans to a deficiency for the region, these lines were somewhat weaker than $h l^{3987}$ hemizygotes (Tables 1 and 2), suggesting that these alleles are also hypomorphs.

To generate additional alleles for further study of the hls gene, we excised the $\mathrm{P}\left(1 a c Z, r y^{+}\right)$element in $h l s^{3987}$ using the $\Delta 2-3$ transposase, scoring for loss of the rosy ${ }^{+}$ $\left(\mathrm{ry}^{+}\right)$eye color marker (Robertson et al. 1988). We hoped to obtain less severe lines in which eggs were laid and any embryonic defects might be analyzed, as well as stronger alleles in which the expressivity of the oogenesis phenotypes might be increased. Following two independent transposase-induced excision screens, $280 \mathrm{ry}^{-}$ lines were established. Of these lines, $31 \%$ were fully fertile, demonstrating that the $\mathrm{P}$ element was responsible for the female sterile phenotype. The sterile, imprecise excision lines, however, were all similar to our start- ing allele in phenotype and range of expressivity. Molecular analyses demonstrated that the $\mathrm{P}$ element is inserted within the coding region of the gene (see below), and we speculate that the presence of any extraneous DNA is deleterious. We did recover three excision lines that fall into one lethal complementation group, are uncovered by a deficiency for the region, and are sterile in trans to the starting P allele. Two of these lines, hls ${ }^{\Delta 157}$ and $h l s^{\Delta 139}$, contain deletions of most or all of the $\mathrm{P}$ element and 1-2 kb of the hls gene $3^{\prime}$ to the P element insertion site (Fig. 3A). These lines therefore may be null for hls function. A third lethal line, hls ${ }^{\Delta 125}$, contains a deletion of the $\mathrm{P}$ element and at least $6 \mathrm{~kb} 3^{\prime}$ to the $\mathrm{P}$ element insertion site, and thus may affect additional genes. Examination of the lethal phase of these deletion alleles demonstrated that $h \mathrm{~h}^{\Delta \mathbf{1 2 5}}$ homozygotes die as embryos, whereas $h l s^{\Delta 157}$ and $h l s^{\Delta 139}$ animals die at all stages of development.

\section{Mutations in hls affect oocyte localization} and dorsal eggshell appendage formation

Ovaries from hls ${ }^{3987}, h l s^{3987} / D f, h l s^{E 616} / D f$, and $h l s^{E 653} /$
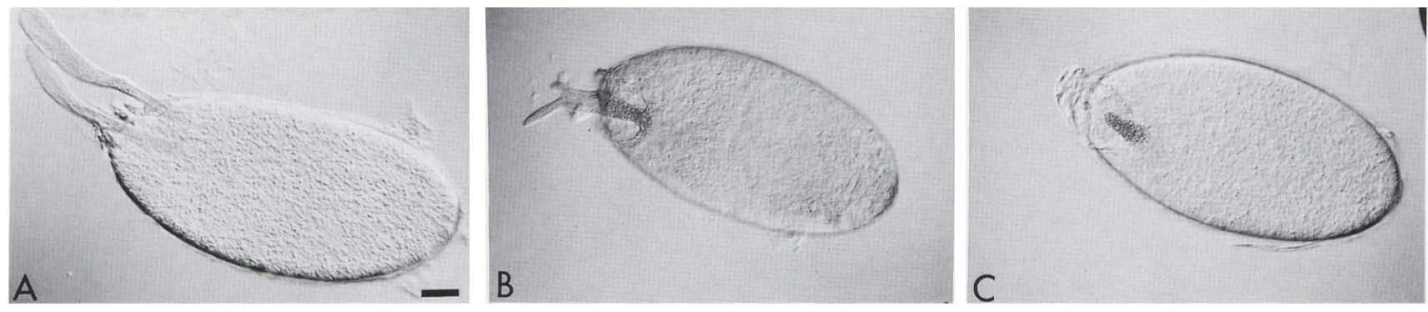

Figure 2. hls egg chambers show a range of ventralized phenotypes. Some wild-type chorion structures are observed, but no eggs hatch from homozygous hls females. The S14 egg chambers shown here were obtained from dissected ovaries, because few eggs are laid. $(A)$ Wild-type S14 egg chamber showing the characteristic dorsolateral respiratory appendages. $(B)$ hls ${ }^{3987}$ S14 egg chamber. The "forked" respiratory appendages arise from a single base on the midline. (C) A more ventralized $h l s^{3987} \mathrm{~S} 14$ egg with a single dorsal appendage. Bar in $A, 20 \mu \mathrm{m}$. 
Table 1. Oocyte mislocalization and early degeneration

\begin{tabular}{|c|c|c|c|}
\hline Genotype & $n$ & $\begin{array}{c}\text { Mislocalized } \\
\text { oocyte }(\%)^{a}\end{array}$ & $\begin{array}{c}\text { Degenerating } \\
\text { S8-S10 } \\
\text { egg chamber } \\
(\%)\end{array}$ \\
\hline \multirow[t]{2}{*}{$h l s^{3987}$} & 123 & $6(5)$ & $7(6)$ \\
\hline & 127 & $9(7)^{b}$ & - \\
\hline$h l s^{3987} / D f(3 R) s b d^{105}$ & 100 & $11(11)$ & $38(38)$ \\
\hline$h 1 s^{E 616} / D f(3 R) s b d^{105}$ & 125 & $7(6)$ & $21(17)$ \\
\hline$h l s^{E 653} / D f(3 R) s b d^{105}$ & 163 & $7(4)$ & $21(13)$ \\
\hline
\end{tabular}

${ }^{a}$ Based on vitellogenic egg chambers $(\mathrm{S} 8-10)$

bBased on oskar hybridization (S1-10)

$D f$ females contained a small percentage of vitellogenic egg chambers with mislocalized oocytes (Fig. 1B; Table 1). To quantify mislocalization in previtellogenic egg chambers, we identified the oocyte by detecting the presence of osk transcripts, which are produced in the nurse cells and then transported to the oocyte early in oogenesis (Fig. 1C; Ephrussi et al. 1991; Kim-Ha et al. 1991). The osk probe recognized transcripts in a single germline cell in hls egg chambers (Fig. 1D). In some egg chambers, the labelled cell was not the posterior-most cell, indicating a mispositioned oocyte (Fig. 1D, arrowheads). In $h l s^{3987}$ homozygotes, $7 \%$ of S1-S10 egg chambers contained mislocalized oocytes (Table 1).

Ovaries dissected from $h l s^{3987}$ females also contained a range of late-stage phenotypes (Table 2). A wild-type egg chamber at $\mathrm{S} 14$ possesses two dorsal eggshell respiratory appendages, just lateral to the dorsal midline (Fig. 2A). Some wild-type-appearing egg chambers were recovered from $h l s^{3987}$ females, but $90 \%-95 \%$ of the egg chambers showed aberrant appendage formation; the majority possessed only one appendage or fused appendages emerging from one base on the dorsal midline /Fig. 2B,C; Table 2). Females transheterozygous for either $h l s^{E 616}$ or $h l s^{E 653}$ and $D f(3 R) s b d^{105}$ produced egg chambers with a similar range of phenotypes (Table 2).

Cloning of the hls gene

To characterize the hls gene at the molecular level, DNA flanking the $5^{\prime}$ end of the $\mathrm{P}$ element was cloned by screening a $\lambda$ library of $h l s^{3987}$ DNA with a probe complementary to the P element $5^{\prime}$ end. The $1.5 \mathrm{~kb}$ of flanking DNA obtained in this way was used to isolate two cosmids from a cosPneo library (gift of Mark Champe, Genentech, San Francisco, CA) (Fig. 3A). In addition, the $1.5-\mathrm{kb}$ flanking DNA hybridized to two abundant transcripts on a Northern blot of wild-type female RNA. A 1.1 -kb transcript showed no variation in length or expression levels between wild-type and $h l s^{3987}$ females (data not shown). Partial sequence of genomic DNA encoding the $1.1-\mathrm{kb}$ transcript revealed strong homology (84\% identity) to bovine $\mathrm{NADH}$ dehydrogenase $(\mathrm{H}$. Enderlin, D. Gillespie, and C. Berg, unpubl.). A second, 4.6-kb transcript was abundant in wild-type females but nearly absent in $h l s^{3987}$ females. We refer to the $4.6-\mathrm{kb}$ transcript as the hls transcript. A subclone specific to the hls transcript was used to isolate a $4.6-\mathrm{kb}$ cDNA from an ovarian cDNA library (Stroumbakis et al. 1994). When this cDNA was used as a probe against Northern blots (Fig. 3B), it recognized the same $4.6-\mathrm{kb}$ transcript as well as an additional, much less abundant, $11-\mathrm{kb}$ transcript whose expression pattern paralleled that of the hls transcript. This additional message may represent either a pre-mRNA for the hls transcript or an alternatively spliced message. Both the 4.6- and 11-kb transcripts were expressed primarily in ovarian tissue, although small amounts were detected in total male and in female carcass RNA. The cDNA also hybridized to a rare, 9-kb transcript, expressed at extremely low levels in ovaries and at moderate levels in male and female carcass tissues. This transcript was unaffected by the P element

Table 2. S14 respiratory appendage formation in hls ovaries

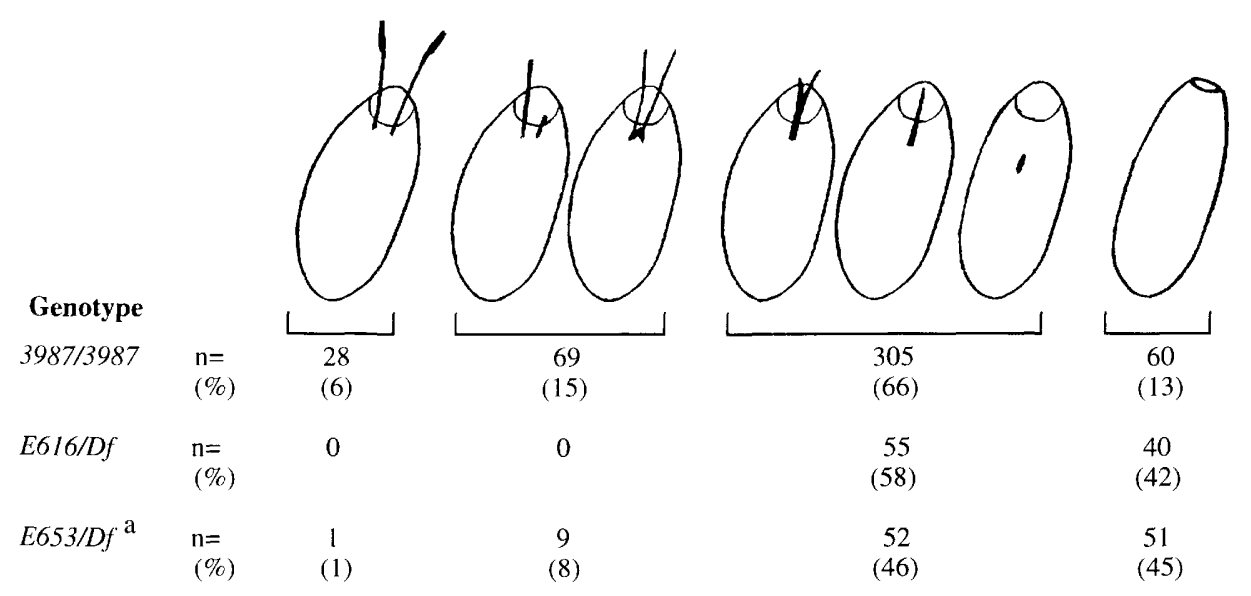

Numbers are from ovaries dissected from 2 to 5-day-old females.

${ }^{a}$ We also observed variable cup and dumpless phenotypes in some females dissected from this genotype. 


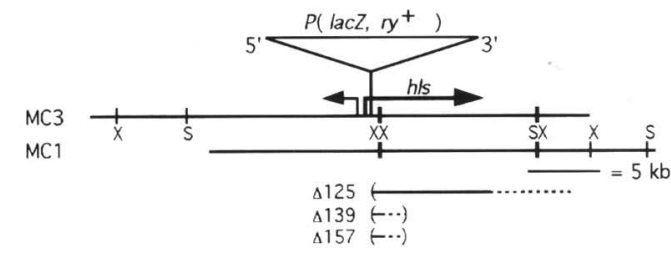

B

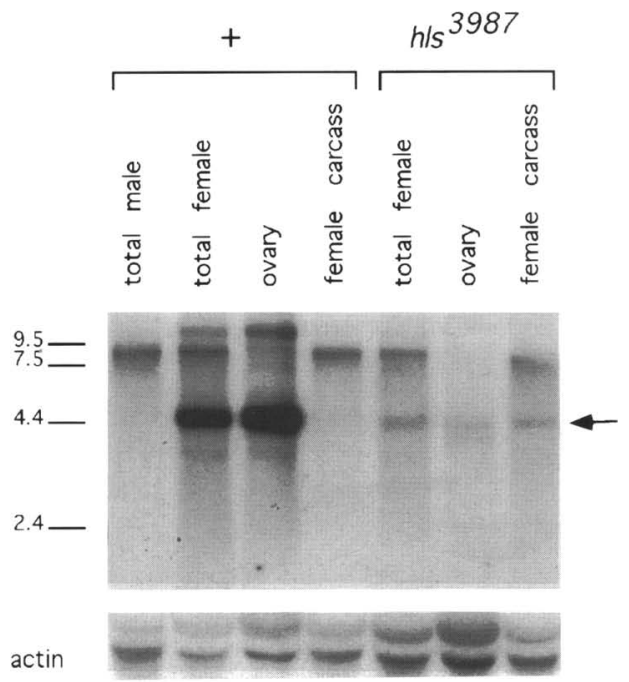

C

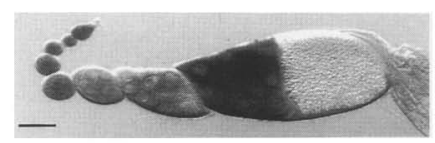

D

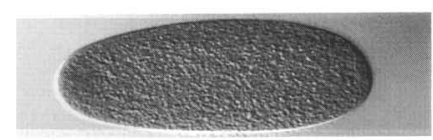

Figure 3. (A) Molecular map of the hls locus. Cosmids MC1 and MC3 were isolated by using a probe from DNA flanking the 5 ' end of the $P$ element (see text). The $4.6-\mathrm{kb}$ hls transcript is indicated as the large arrow pointing to the right. The smaller arrow pointing to the left represents a transcript encoding a protein highly homologous to NADH dehydrogenase. The deletions present in $h l s^{\mathbf{4 1 2 5}}, h l s^{\mathbf{4 1 3 9}}$, and $h l^{\Delta \mathbf{1 5 7}}$ are shown below the map as thin lines bounded by parentheses. The solid lines indicate DNA that is known to be deleted; dotted lines indicate DNA that may be deleted. (X) XbaI; (S) SalI. $\langle B|$ Northern blot probed with the 4.6-kb hls cDNA (cD5). Total RNAs $(35 \mu \mathrm{g})$ were loaded in each lane. The 4.6-kb hls transcript (arrow) is expressed predominantly in females and is enriched in ovarian RNA. This transcript is nearly absent in RNA from $h l s^{3987}$ homozygous females. $(C)$ The same blot was stripped and reprobed to identify actin mRNA as a control to show the amount of RNA present in each lane. $(C, D)$ In situ hybridization of wildtype tissue using the hls cDNA as a probe. $(C)$ Ovariole, germarium through S10. The transcript is first detected in the germarium, falling off in the nurse cells through S4-S5, then increasing in S8, and strongest in S10. The message is transported into the oocyte during S11 and is not localized within the embryo. $(D)$ Preblastoderm stage embryo. Bar in C, $20 \mu \mathrm{m}$.

insertion (Fig. 3B). The lethality of the deletion alleles could be attributable to the lack of carcass transcripts.
To determine the spatial pattern of hls expression, digoxigenin-labeled cDNA sequences were used to probe wild-type ovaries by in situ hybridization. In the germarium, hybridization appeared to be limited to the germline cells (Fig. 3D). hls expression decreased through S4/ $\mathrm{S} 5$, increased during $\mathrm{S} 8$, and was strongest in the nurse cells at $\mathrm{S} 10$ (Fig. 3D). No transcript was detected in the oocyte until S11, when the nurse cell contents are deposited into the oocyte. The hls transcript was distributed uniformly in the early embryo (Fig. 3E).

hls encodes a putative member of the DE-H family of RNA-dependent ATPases/helicases

The sequence of the hls cDNA (Fig. 4) indicated a coding region of 4377 nucleotides, predicting a protein of 1459 amino acids having a molecular mass of $\sim 155 \mathrm{kD}$. The carboxy-terminal two-thirds of the predicted protein revealed no homology in searches using the BLAST and GCG programs. The amino-terminal sequence, however, contained a region similar to yeast splicing factors PRP2 (pre-mRNA processing protein-2) (Chen and Lin 1990) and PRP16 (Burgess et al. 1990) and to the Drosophila Maleless (Mle) protein, which is required for dosage compensation (Kuroda et al. 1991) (underlined region in Fig. 4; Fig. 5). These proteins are members of a large superfamily of RNA-dependent ATPases and helicases, which contain seven broadly defined domains (Hodgman 1988). Within these seven domains, Hls shares $\sim 29 \%$ identity and $51 \%$ similarity with PRP2, PRP16, and Mle. As indicated in Figure 5, domain I contains an ATP/GTPbinding motif.

\section{Hls plays a role in localizing RNAs}

The similarity to proteins that function through binding RNAs suggested a possible role for Hls in RNA processing, transport, or stabilization. Therefore, we examined the localization patterns of seven previously characterized mRNAs that are known to be localized during oogenesis. Our work placed these transcripts into three classes: (1) those that failed to be transported or localized correctly in some fraction of hls egg chambers, (2) those that were localized correctly but were reduced in amount, and (3) those unaffected in hls mutants.

The first class of messages was identified by examination of the localization patterns of the grk, osk, and bcd mRNAs. Because of the highly penetrant dorsoventral defects in late-stage $h l s$ egg chambers, we suspected that grk expression or localization might be defective. In wild-type oocytes, the $g r k$ transcript is tightly localized to the dorsal anterior cortex, overlying the nucleus in a cap at S9 and S10 (Fig. 6A; Neuman-Silberberg and Schüpbach 1993). Surprisingly, we found that grk mRNA was localized appropriately in the majority of S9 and S10 hls egg chambers (Fig. 6B; Table 3). In $\sim 30 \%(n=52)$ of hls egg chambers, however, the pattern was defective. We observed some eggs with no grk mRNA localization, some S10 eggs with an anterior ring (Fig. 6C), and some 


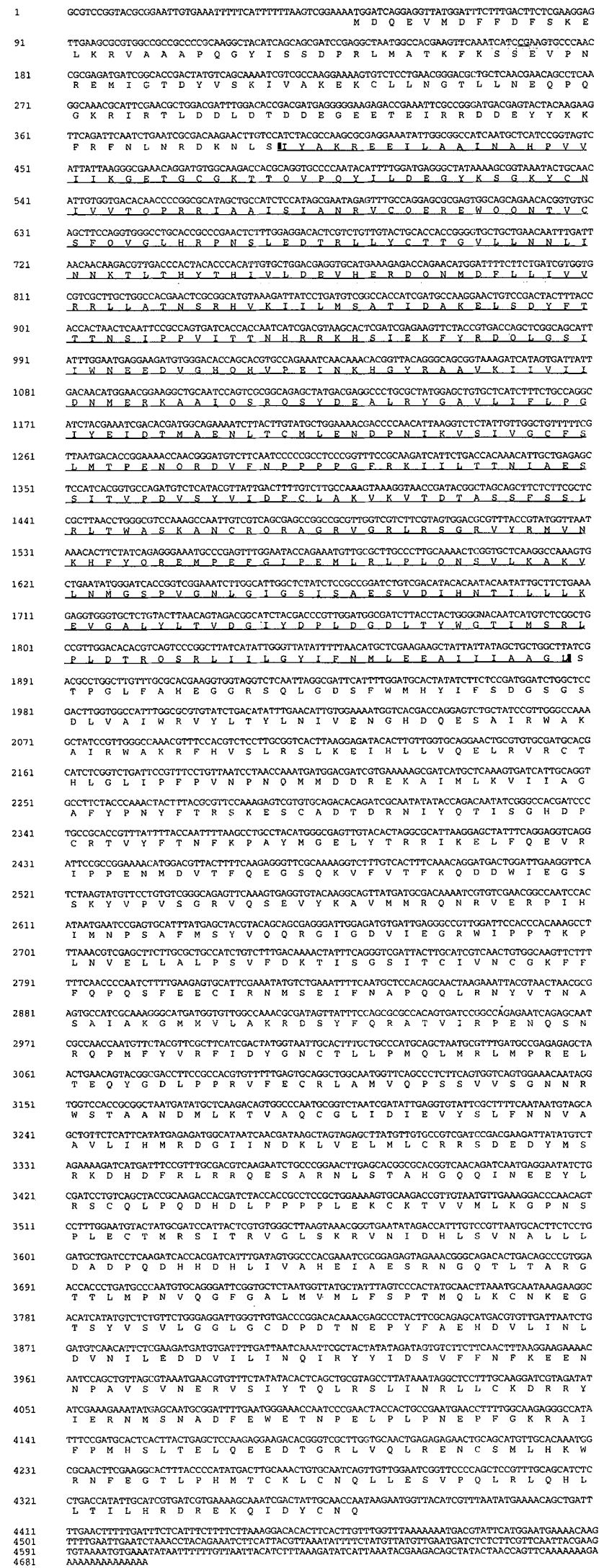

Figure 4. cDNA sequence and predicted protein sequence of hls cDNA. The sequence presented here was generated from a single, full-length cDNA (cD5). The numbers correspond to DNA base sequence. The region aligned for homology comparison in Fig. 5 is underlined. The 2 bases between which the P element is inserted in $h l s^{3987}$ (nucleotides 168 and 169) are underlined.

with a dorsal patch that was broader than normal (Table 3).

As described above, we used in situ hybridization with an osk cDNA probe to identify the previtellogenic oocytes in hls ovaries. Although osk RNA was transported to the oocyte normally in these early stages, later localization to the posterior pole was defective. In the majority of S10 egg chambers, osk RNA was diffuse throughout the oocyte (Fig. 6E). In those egg chambers in which osk RNA was not distributed uniformly, only $12 \%(n=77)$ contained osk message correctly localized to the posterior pole. More frequently, we observed a diffuse accumulation of osk RNA in the center or toward the anterior of the oocyte (Fig. 6F; Table 3). About half of the eggs within this latter class also had a small amount of osk mRNA localized to the posterior pole (Fig. 6F).

Bcd is an anterior determinant whose transcript is lo-

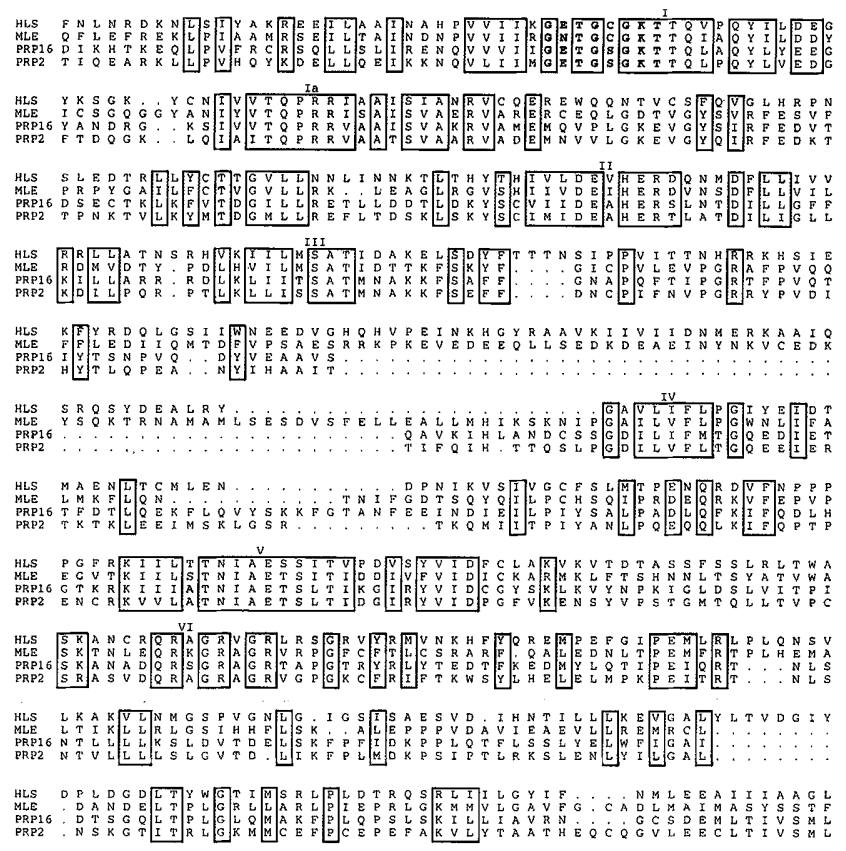

Figure 5. Alignment of the amino-terminal region of Hls with three DE-H family members. Hls amino acids 116-623 are aligned with Drosophila Mle and yeast PRP2 and PRP16. Identi$\mathrm{cal}$ and similar amino acids are boxed if represented in all four proteins. Acceptable similarities were as follows: $[\mathrm{V}, \mathrm{I}, \mathrm{L}, \mathrm{M}]_{\text {; }}$ $[F, Y, W]_{;}[R, K] ;[D, E] ;[T, S]$. Roman numerals indicate the seven motifs originally identified by Hodgman (1988) and found in the DEAD/DE-H superfamily. No definitive consensus sequences or boundaries for each motif have been described in the literature. The ATP/GTP-binding domain is indicated in bold. 

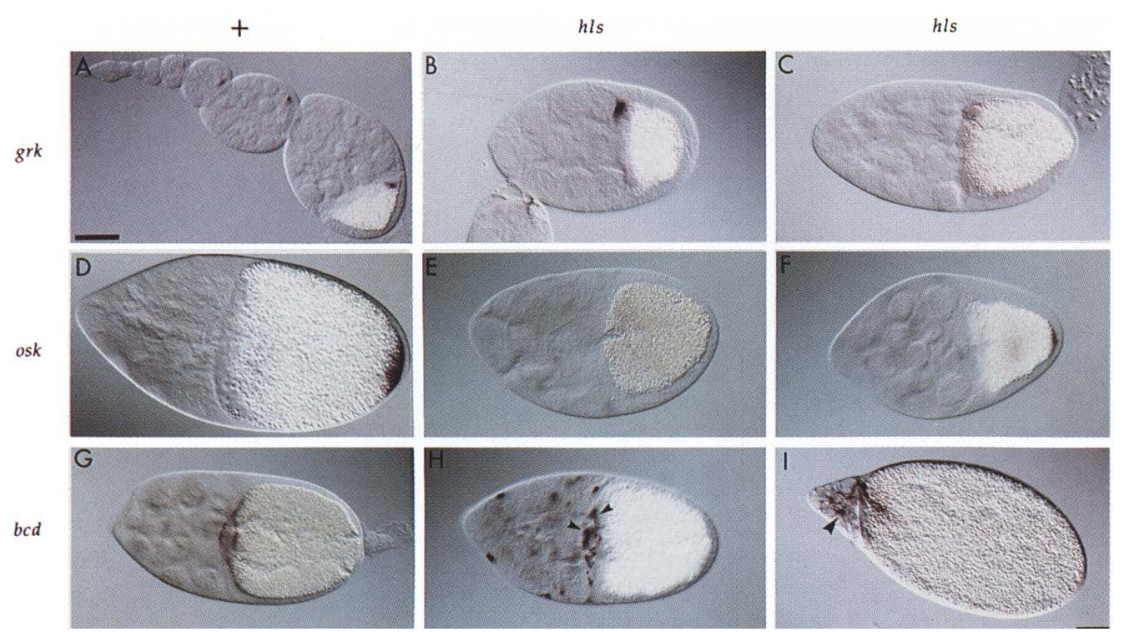

Figure 6. Wild-type and $h l s^{3987}$ egg chambers, probed with grk, osk, and bcd sequences. (A) Wild-type ovariole, probed with grk sequences. $(B, C) h l s^{3987}$ egg chambers, probed with grk sequences. grk message is localized correctly in the majority of $\mathrm{S} 10$ hls egg chambers $(B)$, but in some $\mathrm{S} 10$ egg chambers, grk transcript is absent or localized in a faint anterior ring $(C) .(D) \mathrm{S} 10$ wild-type egg chamber, probed with osk sequences. $(E, F) h l s^{3987}$ egg chambers, probed with osk sequences. In the majority of S10 $h l s$ egg chambers $(E)$, osk message is diffuse throughout the oocyte. Occasionally, osk mRNA accumulates near the anterior or in the center of the oocyte, frequently coupled with a small amount of osk transcript at the posterior pole, shown here $(F)$ in a $S 9$ egg chamber. $(G)$ S10 wild-type egg chamber, probed with $b c d$ sequences. $(H, I) h l s^{3987}$ egg chambers, probed with bcd sequences. bcd message is detected in a punctate pattern in $\mathrm{S} 10 \mathrm{hls}$ egg chambers, and the majority of the message is retained in the nurse cells $\langle H\rangle$. Arrowheads $(H)$ mark clusters of $b c d$ transcript that fail to move laterally to the outer cortex. In S11 wild-type egg chambers, no bcd transcript is detected in the nurse cells (data not shown), whereas nurse cells in S11 hls egg chambers $(I)$ retain significant amounts of $b c d$ message (arrowhead). The bar in $A$ applies to $A-H$ and is $20 \mu \mathrm{m}$; Bar in $I$, also $20 \mu \mathrm{m}$.

calized to the anterior cortex of the oocyte during S8-S10 of oogenesis (Fig. 6G; Berleth et al. 1988; St. Johnston et al. 1989). The mRNA is produced in the nurse cells where it transiently accumulates at the apical cortex before being transported to the oocyte (Berleth et al. 1988; St. Johnston et al. 1989). In hls egg chambers, we observed clearly aberrant $b c d$ mRNA distribution in S8. In most egg chambers (Fig. $6 \mathrm{H}$; Table 3 ), some $b c d$ message was transported to the oocyte, but the majority remained in the nurse cells, concentrated at the apical cortex. In addition, much of the $b c d$ message within the oocyte was not transported laterally toward the periphery but instead remained centrally located, possibly at the site of the ring canals that connect the nurse cells to the oocyte (Fig. 6H, arrowheads). Even during the normal bulk transport of material from the nurse cells to the oocyte at S11, some bcd message still remained in the nurse cells (Fig. 6I, arrowhead). The failure to transport $b c d$ mRNA to the oocyte and osk message within the oocyte during S8-S10 are the earliest defects in RNA transport we observe in hls mutants and clearly indicate that normal hls functions are disrupted at the beginning of vitellogenesis.

Examination of two other anteriorly localized tran-

Table 3. Localization of $m$ RNAs in $S 10$ egg chambers

\begin{tabular}{|c|c|c|c|}
\hline mRNA & Wild type & $h l s^{3987}$ & $\begin{array}{c}\text { Percent } \\
\text { egg chambers }{ }^{a}\end{array}$ \\
\hline \multirow[t]{4}{*}{ gurken } & dorsal anterior & dorsal anterior & 68 \\
\hline & & broader dorsal patch & 14 \\
\hline & & anterior ring & 13 \\
\hline & & no localization & 5 \\
\hline \multirow[t]{4}{*}{ oskar } & posterior pole & posterior pole & 12 \\
\hline & & anterior or central cloud, plus some at posterior & 14 \\
\hline & & anterior or central cloud only & 14 \\
\hline & & diffuse throughout the oocyte & 60 \\
\hline \multirow[t]{3}{*}{ bicoid } & anterior cortex & majority at anterior of oocyte, plus some in nurse cells & 10 \\
\hline & & clumps at anterior of oocyte and punctate in nurse cells & 74 \\
\hline & & none in oocyte and punctate in nurse cells & 16 \\
\hline K10 & anterior & anterior, reduced amount & $100^{\mathrm{b}}$ \\
\hline orb & anterior & anterior, reduced amount & $100^{\mathrm{b}}$ \\
\hline Bicaudal-D & anterior cortex & anterior cortex & 100 \\
\hline hu-li tai shao & anterior cortex & anterior cortex & 100 \\
\hline
\end{tabular}

The localization patterns in previtellogenic stages were similar in wild-type and hls egg chambers for all transcripts listed. agurken, $n=52$; oskar, $n=77$; bicoid, $n=49 ; k 10, n=50$; orb, $n=79$; Bicaudal- $D, n=127$; hu-li tai shao, $n=42$.

${ }^{\mathrm{b}}$ Although proper localization was observed in $100 \%$ of egg chambers, transcript levels were reduced. 
scripts, the $K 10$ and orb mRNAs, identified a second class of messages. K10, which is required for correct grk mRNA localization and dorsoventral axis formation (Wieschaus et al. 1978; Neuman-Silberberg and Schüpbach 1993), is transcribed in the nurse cells. The message is transported to the oocyte during previtellogenic stages (Cheung et al. 1992; Fig. 7A). At the onset of vitellogenesis (S8), the transcripts are localized to the anterior edge of the oocyte where they accumulate through S10 (Fig. 7B). In a hls ${ }^{3987}$ background, K10 transcripts were transported to the oocyte and later localized to the anterior edge (Fig. $7 \mathrm{C}$ ), but the level of transcript was greatly decreased relative to wild type, specifically in vitellogenic stages (Fig. 7D).

Our observations using an orb cDNA probe were similar to those with $K 10$. The orb gene encodes sexspecific RNA-binding proteins (Lantz et al. 1992) required for correct osk and grk mRNA localization (Christerson and McKearin 1994). In wild-type ovarioles, orb transcript is transported to the oocyte in the germarium and continues to accumulate in the oocyte through previtellogenic stages (Fig. 7E). At S8, the transcript is localized to the anterior edge of the oocyte, but unlike $K 10$, the intensity of the anterior band decreases through S10 (Fig 7F; Lantz et al. 1992). In $h l s^{3987}$ egg chambers, orb mRNA accumulated normally in the oocytes of early egg chambers (Fig. 7G), but the anterior localization, while present, was significantly weaker in S8-S10 (Fig. $7 \mathrm{H})$.

We found that the transport and localization patterns of the Bic-D and hts mRNAs did not change in $h l s^{3987}$ ovaries. Following early oocyte accumulation, the Bic-D transcript is localized to the anterior of the oocyte at S8 (Fig. 7I). This anterior localization is maintained through $\mathrm{S} 10$, at which time nurse cell expression of Bic- $D$ increases dramatically (Fig. 7J; Suter et al. 1989). hts transcripts are also present in low levels in wild-type germaria and are transported to the oocyte from the nurse cells (Yue and Spradling 1992; Ding et al. 1993b). During vitellogenic stages, $h$ ts transcripts are localized predominantly to the anterior of the oocyte (Fig. 7M) and remain localized during nurse cell dumping at S11 (Fig. $7 \mathrm{~N}$ ).
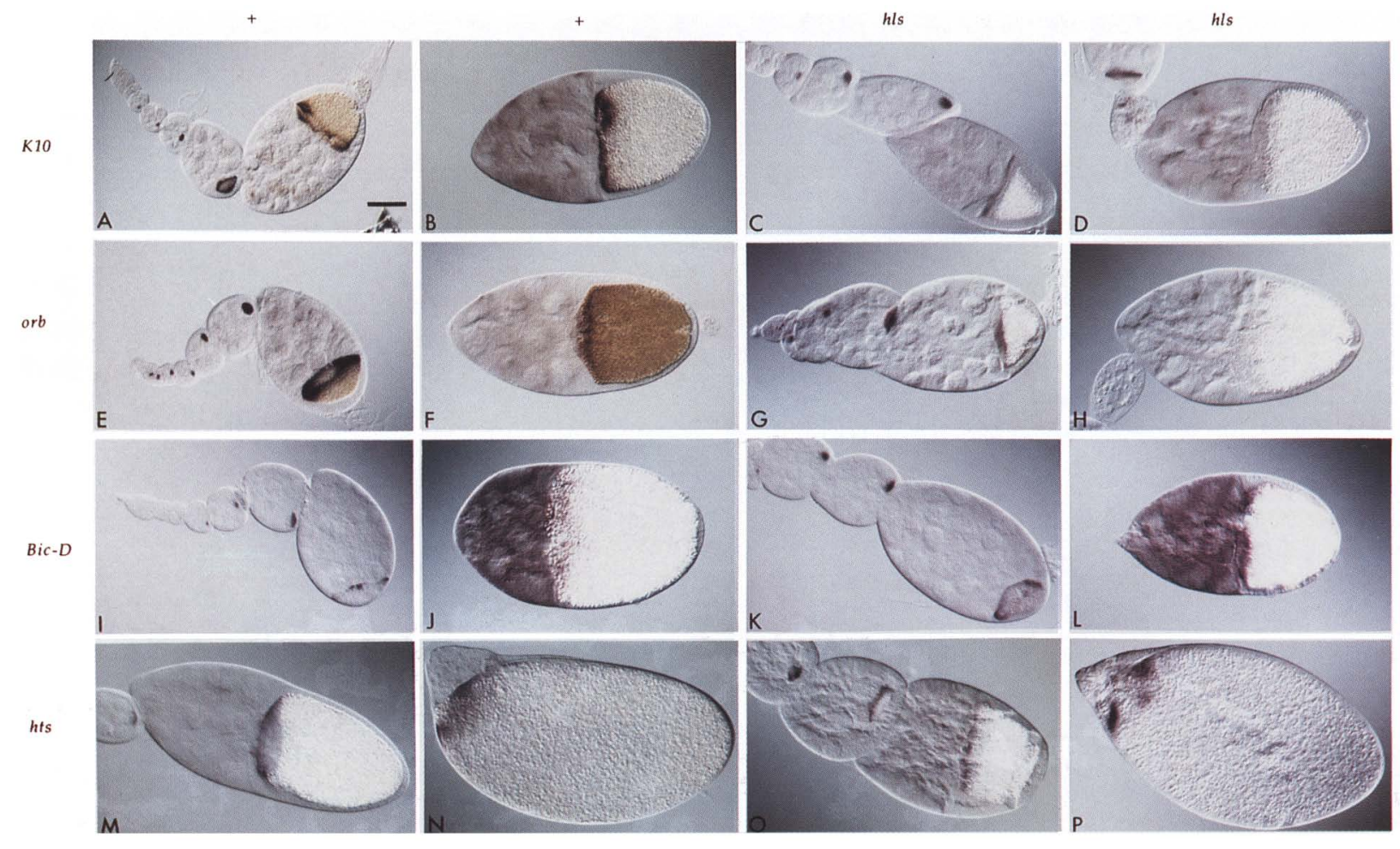

Figure 7. Wild-type and $h l s^{3987}$ egg chambers, probed with $K 10$, orb, Bic-D, and hts sequences. $(A, B)$ Wild-type egg chambers, probed with $K 10$ sequences. $(C, D) h 1 s^{3987}$ egg chambers, probed with $K 10$ sequences. Localization of $K 10$ message to the oocyte is normal during early oogenesis $(C)$. Anterior localization of $K 10$ transcript occurs normally in $\mathrm{S} 10 \mathrm{hls}$ egg chambers $(D)$, although the amount is reduced $(D)$ compared to wild type $(B) .\{E, F\rangle$ Wild-type egg chambers, probed with orb sequences. $(G, H) h 1 s^{3987}$ egg chambers, probed with orb sequences. orb message is transported normally to the oocyte in hls egg chambers during early oogenesis $(G)$. Anterior localization is normal in S10 hls egg chambers, but the level is reduced $(H)$. (I,,$)$ Wild-type egg chambers, probed with Bic-D sequences. $(K, L) h l s^{3987}$ egg chambers, probed with Bic-D sequences. Early transport to the oocyte and anterior localization within the oocyte is observed in both wild-type $(I)$ and $h I s(K)$ ovarioles. Transcription of Bic-D increases dramatically during S9 and S10, and message within the oocyte is anteriorly localized in both wild-type $(J)$ and $h l s$ egg chambers $(L)$. $(M, N)$ Wild-type egg chambers, probed with $h t s$ sequences. $(O, P) h l s^{3987}$ egg chambers, probed with hts sequences. Localization of hts message during all stages of oogenesis $(O, P)$ is normal in hls egg chambers. Bar in $A, 20 \mu \mathrm{m}$. 

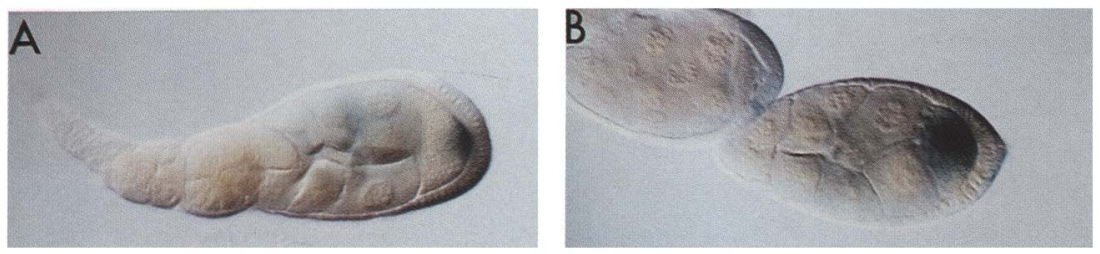

Figure 8. Kin: $\beta$-Gal localization in wildtype and hls egg chambers. (A) S8 wild-type egg chamber. At $\mathrm{S} 8$, Kinesin-directed $\beta$-Galactosidase activity is observed at the posterior of the oocyte. (B) S8 hls ${ }^{3987}$ egg chamber. $\beta-G a l$ activity indicates that the fusion protein is diffuse throughout most of the oocyte.

These patterns were not affected by our $h l s^{3987}$ mutation (Fig. $7 \mathrm{~K}, \mathrm{~L}, \mathrm{O}, \mathrm{P}$ ).

\section{Microtubule structure is disrupted in hls egg chambers}

Because the Hls protein has homology to RNA-binding proteins of the DE-H family, it seemed likely that it was acting directly to localize RNAs in oogenesis. It was possible, however, that mutations in the gene were affecting RNA localization by disrupting the microtubule architecture that is a common component of the localization mechanisms of these RNAs (Koch and Spitzer 1983; Pokrywka and Stephenson 1991, 1995; Theurkauf et al. 1993; for review, see Theurkauf 1994a). In addition, the diffuse osk mRNA pattern that we observed in hls egg chambers has been described in mutants affecting microtubule organization (Ephrussi et al. 1991; Kim-Ha et al. 1991; Ruohola et al. 1991; Clark et al. 1994; GonzálezReyes and St. Johnston 1994; Lane and Kalderon 1994; Theurkauf 1994b; Ganzález-Reyes et al. 1995; Roth et al. 1995). Hence, we examined the localization of a Kinesin heavy chain: $\beta$-Galactosidase (Kin: $\beta$-Gal) fusion protein (Clark et al. 1994) in a $h l s^{3987}$ background to analyze one aspect of microtubule structure and function. In wild-type S8 and S9 egg chambers, microtubule organizing centers (MTOCs) are located at the anterior of the oocyte and direct the formation of a gradient of microtubules whose plus ends extend toward the posterior pole (Theurkauf et al. 1992). The fusion protein is thus propelled by the plus-end-directed kinesin motor function and is present in a band at the posterior of the oocyte, as detected by $\beta$-Galactosidase activity on an $\mathrm{X}$-Gal substrate (Fig. 8A). In a hls background, we detected the fusion protein in the center of S8 oocytes and in the central and lateral portions of $\mathrm{S} 9$ oocytes (Fig. 8B), suggesting that microtubule structure or function is disrupted.

To verify our results using the Kin: $\beta$-Gal fusion protein, we directly examined microtubule organization in hls egg chambers by comparing anti- $\alpha$-Tubulin immunofluorescence in wild-type and $h l s^{3987}$ ovaries. An accumulation of microtubules, indicating the MTOC, is observed at the posterior of the oocyte prior to vitellogenesis in wild-type egg chambers (Fig. 9A; Theurkauf et al. 1992). At the onset of vitellogenesis in S8, the MTOC is no longer present at the posterior, having moved anteriorly along the cortex (Fig. 9C,E). This anterior cortical accumulation is still apparent during S9 (Fig. 9G) and persists through S10a. The oocyte nucleus moves anteriorly with the MTOC at S8 (Theurkauf et al. 1992). In hls egg
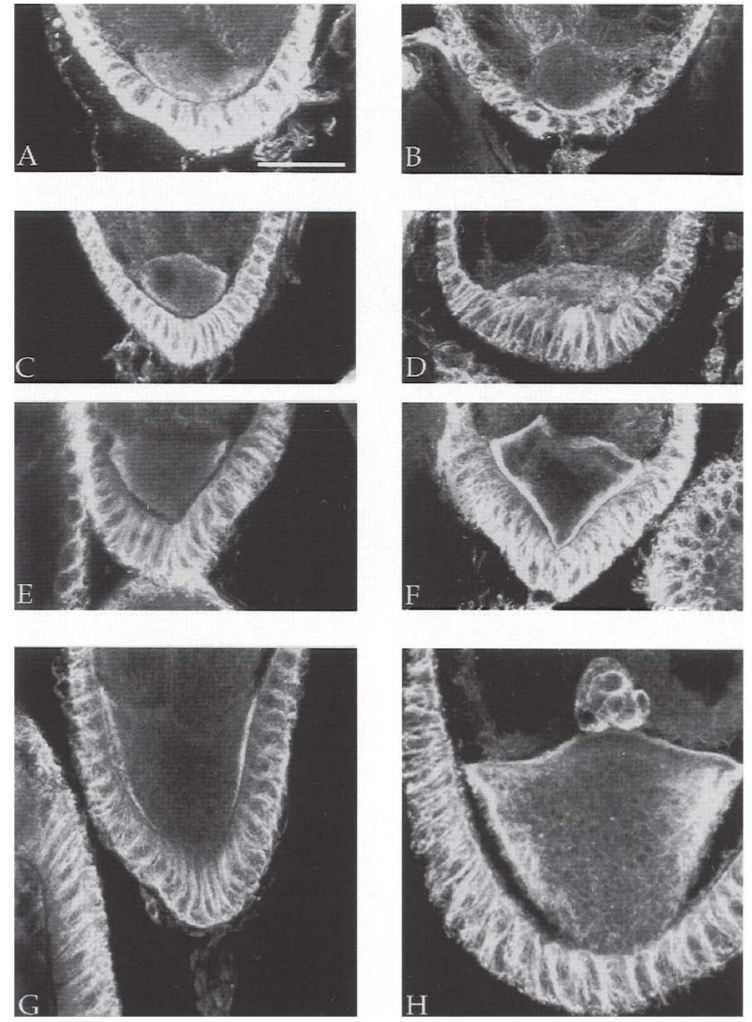

Figure 9. Microtubule organization in wild-type and $h l s^{3987}$ egg chambers. We visualized microtubule structure by immunofluorescence using anti- $\alpha$-Tubulin antibodies. Each image is a projection of three confocal images, $1 \mu \mathrm{m}$ apart. Posterior is toward the bottom. $(A, C, E, G)$ Wild-type oocytes; $(B, D, F, H)$ $h l s^{3987}$ oocytes. In $S 7$ oocytes from wild-type $(A)$ and $h l s(B)$ egg chambers, we observed similar posterior accumulation of microtubules. Cortical microtubules move anteriorly in early wild-type $\mathrm{S} 8$ oocytes $(C)$ and are absent at the posterior by late S8 (E). Two main phenotypes were observed in hls egg chambers. Over half of hls 88 oocytes contained long internal microtubule bundles $(D)$; in the remaining oocytes, cortical accumulation was present at the anterior but also persisted at the posterior $(F)$. In wild-type S9 oocytes, little or no internal microtubule meshwork was detected $(G)$, whereas hls S9 oocytes contained extensive microtubules initiating at the cortices and extending into the cytoplasm centrally and posteriorly $(H)$. This image also illustrates the premature arrival of the border cells at the nurse cell/oocyte boundary. The membrane separation observed in this image was a result of dehydration during methanol fixation and was observed in both wild-type and hls egg chambers. 
chambers, we observed a normal posterior concentration of microtubules prior to vitellogenesis (Fig. 9B). In contrast, a dense network of microtubules was present throughout the oocyte in $64 \%(n=55)$ of the S8 hls egg chambers (Fig. 9D). In the remainder of the 58 egg chambers, cortical microtubule accumulation was observed both posteriorly and anteriorly (Fig. 9F). hls S9 egg chambers contained an extensive microtubule mesh concentrated at the anterior cortices $(72 \%, n=14)$. Unlike wild type, this mesh clearly extended centrally and posteriorly into the cytoplasm. In all cases, the oocyte nucleus was correctly positioned at the dorsal anterior corner of the oocyte.

During S10b, the oocyte microtubules reorganize and begin cytoplasmic streaming in preparation for the transport of cytoplasm from the nurse cells (Gutzeit and Koppa 1982; Theurkauf et al. 1992). Despite the presence of an aberrant network in S8-S9 hls egg chambers, we observed normal microtubule rearrangements in S10b hls oocytes (data not shown). Time-lapse photography also indicated that temporal regulation of streaming in hls egg chambers is identical to that in wild type /data not shown). Thus, the observed defects in RNA and Kin: $\beta$-Gal localization in hls egg chambers are not attributable to premature cytoplasmic streaming but, rather, to the inappropriate formation of an extensive microtubule meshwork during S8-S9.

\section{Discussion}

The hls gene is required for proper localization of several RNAs that are involved in anteroposterior and dorsoventral axis formation during oogenesis. Based on the RNA localization patterns and microtubule structure in hls mutants, and the similarity of Hls protein to members of the DE-H family of RNA-binding proteins, we present models for the role of Hls in oogenesis.

\section{Hls contains homology to RNA-dependent ATPases}

Sequence analysis revealed that the predicted amino-terminal region of the Hls protein is similar to the DE-H family of RNA-dependent ATPases. Although literature citations frequently refer to members of this family as helicases, only some DE-H proteins have demonstrated helicase activity. For example, human RNA Helicase A and vaccinia virus NPH-II have exhibited the ability to unwind double-stranded RNA (Shuman 1992; Lee and Hurwitz 1993). In contrast, two of the three proteins that share strong similarity with Hls, PRP2 and PRP16, demonstrated no helicase activity when tested (Schwer and Guthrie 1991; Kim et al. 1992). The third protein, Mle, has not been examined, although it contains extensive identity over its entire length to the known helicase human RNA Helicase A. Interestingly, Mle and Hls share a similar structure in that their homologous domains are limited to the amino-terminal third of the proteins. In PRP2, an 876-amino-acid protein, the DEAD/DE-H domain is located centrally in the sequence, similar to the structure of the 1071-amino-acid PRP16.

PRP2 and PRP16 both exhibit nonspecific RNA-bind- ing activity and hydrolyze ATP when single-stranded RNA is provided (Schwer and Guthrie 1991; Kim et al. 1992). PRP2 is required for the $5^{\prime}$ cleavage reaction in splicing (Kim and Lin 1993), and PRP16 for 3' cleavage (Schwer and Guthrie 1991). Mle is implicated in dosage compensation in Drosophila (Belote and Lucchesi 1980) and binds predominantly to the $\mathrm{X}$ chromosome in males (Kuroda et al. 1991). The identity of the bound substrates and any biochemical activities of Mle have not been established. Kuroda et al. (1991) propose models in which Mle is involved in transcript elongation, release of the nascent RNA from the template, or increasing RNA processing efficiencies.

Vasa, a member of the DEAD/DE-H superfamily (Lasko and Ashburner 1988), is required to establish a/p polarity during Drosophila oogenesis. This DEAD-box protein is necessary for the localization of nanos transcripts to the posterior pole during oogenesis. Hls is the first member of the DE-H-box family implicated in RNA transport and localization. Whereas the sequence similarity suggests that Hls interacts directly with RNA molecules, the defects in cytoskeletal organization argue that Hls functions indirectly in localizing specific RNAs required for embryonic polarity. How does hls fit in to the known pathways required for RNA localization?

\section{hls is unique among patterning mutants}

Over the last two decades, a large number of genes required during Drosophila oogenesis for proper localization of RNAs involved in a/p or d/v patterning have been identified. In addition, the dynamic patterns of cytoskeletal organization have been characterized, and requirements for various cytoskeletal components in axis formation have been defined. The hls gene appears to be a link between patterning genes and microtubule architecture.

The mispositioned oocyte and ventralized late-stage egg chamber phenotypes of hls ovaries bear a striking resemblance to some defects observed in members of the spindle class of maternal effect mutants (Tearle and Nüsslein-Volhard 1987). In spn-C mutants, for example, Kin: $\beta$-Gal movement and the localization of some RNAs are disrupted in dicephalic egg chambers, but not in spn-C egg chambers in which the oocyte is positioned normally at the posterior of the egg chamber (GonzálezReyes and St. Johnston 1994). These results suggest that the positioning of the oocyte within the egg chamber directs the formation of the microtubule network within the egg and in this way affects the localization of both RNA and protein products. hls differs from these mutants in that Kin: $\beta$-Gal and the various RNAs are also mislocalized in egg chambers of normal polarity.

The female sterile genes cappucino and spire are thought to regulate the microtubule-dependent cytoplasmic streaming that occurs in S10B-S12 oocytes (Theurkauf 1994b). Mutations in these genes result in the mislocalization of several RNAs and proteins, including osk, K10, and grk mRNAs and Kin: $\beta-G a l$ and Stau proteins (Ephrussi et al. 1991; Kim-Ha et al. 1991; St. Johnston et 
al. 1991; Cheung et al. 1992; Neuman-Silberberg and Schüpbach 1993; Clark et al. 1994). Although these mutants are similar to hls in their variability and range of phenotypes, cytoplasmic streaming initiates normally in hls egg chambers. In addition, cappucino and spire differ from $h l s$ in that they do not disrupt localization of $b c d$ mRNA in the nurse cells or in the oocyte.

The phenotypes of hls mutants most closely resemble those observed in Notch $(N)$, protein kinase A $(P K A)$, grk, top, and cni mutants (Ruohola et al. 1991; Lane and Kalderon 1994; González-Reyes et al. 1995; Roth et al. 1995). Mutations in these genes also affect microtubule structures, but in these cases by disrupting signal transduction pathways required for normal microtubule reorganization within the oocyte during S7-S9. In egg chambers from these mutants, Kin: $\beta-\mathrm{Gal}$ is centrally located in the oocyte, suggesting that microtubules are organized with their positive ends toward the center. osk RNA is also found in the center, whereas bcd message appears at both anterior and posterior poles. Although Hls may be required in these pathways to direct the microtubule changes that occur during mid-oogenesis, it must also play an additional role in $b c d$ mRNA transport from nurse cells to oocyte. Unlike the observations made with $N, P K A$, grk, top, and cni mutants, bcd message is never detected at the posterior of hls egg chambers, even in those few egg chambers in which bcd mRNA is present in the oocyte. The retention of bcd message in nurse cells is not due to a failure in general transport, as the bulk of nurse cell cytoplasm flows normally into the oocyte at S11. Thus, the bcd transcript must be retained because of a specific defect in cytoskeletal architecture or the absence of some critical component in the transport process.

\section{Models for hls function}

From our analyses, we conclude that mutations in hls disrupt microtubule organization at the onset of vitellogenesis, subsequently affecting the transport and localization of selected mRNAs. The expression of hls during early oogenesis, the early phenotype of mislocalized oocytes, and the subsequent degeneration during all stages indicate that $\mathrm{Hls}$ also functions during early stages. Construction of germ-line clones with lethal excision lines may enhance the penetrance of the early defects and reveal an essential requirement for $\mathrm{Hls}$ in previtellogenic egg chambers, perhaps in oocyte specification, microtubule organization, or transport of transcripts to the oocyte. The strong mutant phenotypes observed in the hypomorphic alleles suggest that Hls acts predominantly during vitellogenic stages, and this study has focused on the role of hls during those stages.

We postulate that Hls could be functioning in one of two ways. First, Hls could act downstream of the signaling pathways that induce correct microtubule reorganization during S8 and S9, actively interacting in microtubule reorganization. A second possibility is that Hls is required for efficient transcription, pre-mRNA processing, localization, or translational regulation of products that control the kinetics of microtubule assembly or di- rect the reorganization of microtubule structures. The similarity of Hls protein to members of the DE-H family makes this hypothesis attractive.

To determine the function of hls in axis formation during oogenesis, we examined the distribution of seven different mRNAs in hls ovaries. These analyses coupled with observations of microtubule structures permit categorization of these transcripts by their dependence on properly organized microtubules for localization within the oocyte. Transport of osk and bcd RNAs is highly dependent on a well-organized, polarized network of microtubule arrays interconnecting the nurse cells and oocyte. We observed defective microtubule organization in $78 \%$ of hls S8 and S9 egg chambers, concomitant with mislocalized osk message in $88 \%$ of hls S8-S10 egg chambers. bcd mRNA was affected to a similar degree, with $90 \%$ of hls egg chambers exhibiting significant nurse cell retention of message. Defects in $b c d$ message transport such as those we observe in hls mutants have not been described previously. Our data suggest that microtubule structures undergo a dynamic reorganization in nurse cells during S8, similar to that observed in the oocyte. In hls egg chambers, a lack of reorganization in the nurse cells could result in the abnormal retention of $b c d$ mRNA at the nurse cells cortices.

Despite the severe microtubule defects observed in $h l s$ oocytes, the majority of grk mRNA is localized appropriately, presumably reflecting the correct positioning of the oocyte nucleus. The ventralization of hls egg chambers may be attributable to slightly lower levels of grk message (which we observed but is difficult to quantify), a defect in grk mRNA translation, or to the mislocalization or absence of an as-yet-unidentified transcript or protein. In addition, only a portion of the $K 10$ and orb messages are either mislocalized or degraded in hls oocytes, suggesting that microtubules are moderately necessary for their localization or stabilization at the anterior cortex. In contrast, Bic-D and hts transcripts are independent of the effect of hls mutations on microtubules, being correctly localized in hls egg chambers. These RNAs may be transported and localized when only a subset of the microtubule network is properly formed.

Transport and localization of $b c d$, osk, Bic-D, orb, and K10 mRNAs have been well studied in ovaries treated with colchicine (Pokrywka and Stephenson 1991, 1995). Whereas all of these transcripts are vulnerable to microtubule depolymerization, their dependence on the exact polarization and organization of the cytoskeleton is not well demonstrated by such experiments. Drug treatment data, along with analyses of mutants such as $h l s, N$, and $P K A$, will be necessary to understand fully the microtubule-mediated movement of mRNAs during oogenesis.

These studies provide the first evidence for a DE-H family member acting in processes that direct microtubule organization. Further characterization of the selective effects of hls mutations on specific transcripts will illuminate the different mechanisms for RNA localization and will clarify their relative dependence on the cytoskeleton. 


\section{Materials and methods}

Fly stocks

The wild-type strain employed in our studies was Canton-S. $h l s^{E 616}$ and $h l s^{E 653}$, generous gifts of Daniel St. Johnston, are listed in the Tübingen Stock List as spindle-E mutations (Tearle and Nüsslein-Volhard 1987). $D f(3 R) s b d^{105}$ [88F9-89A1; 89B4-5] was described in Lindsley and Zimm (1992) and was obtained from the Bloomington Stock Center. Jim Kennison (National Institutes of Health, Bethesda, MD) provided additional deficiencies, $D f(3 R) P O^{2-4}, D f(3 R) c 3 G 2, D f(3 R) s b d^{26}$, and $D f(3 R) s b d^{45}$, which were used for more precise mapping of the $\mathrm{P}$ element insertion site. Kinesin heavy chain:lacZ insertion line KL32, kindly provided by $\mathrm{H}$. Ruohola-Baker (University of Washington, Seattle), was used for the Kin: $\beta-$ Gal fusion protein studies. Fly stocks were maintained at $24^{\circ} \mathrm{C}$ on standard cornmeal/yeast/molasses medium.

To generate additional alleles of the hls gene, chromosomes carrying the $\mathrm{P}$ element insertion were first cleaned of potential background mutations in a process that recombined on the visible body color marker ebony $(e)$. Excision lines then were generated by reintroducing the $\Delta 2-3$ transposase (Robertson et al. 1988). Males carrying both transposons were mated in vials to $r y^{506}$ virgins, and the progeny were scored for loss of the ry ${ }^{+}$ eye-color marker. ry ${ }^{-} F_{1}$ males $(280)$ were mated individually to $r y^{506} / T M 3, r y^{R K} S b e^{s}$ virgin females to generate balanced stocks. Flies from each line were crossed to $h l s^{3987} / T M 3$ and $D f(3 R) s b d^{105} / L V M$ flies to test viability and fertility.

\section{Staining and in situ hybridization procedures}

For $\beta$-Gal staining, ovaries were fixed and stained as described by Clark et al. (1994), except that the ovaries were fixed in $0.5 \%$ glutaraldehyde. Micrographs were generated on a Nikon Microphot FXA microscope using differential interference contrast optics.

For in situ hybridization to ovaries, a protocol adapted from Ephrussi et al. (1991) was used. Ovaries were dissected in modified EBR $129 \mathrm{~mm} \mathrm{NaCl}, 4.7 \mathrm{~mm} \mathrm{KCl}, 1.9 \mathrm{~mm} \mathrm{CaCl}, 10 \mathrm{~mm}$ HEPES at $\mathrm{pH} 6.9$; Ephrussi and Beadle 1936) and fixed in $4 \%$ paraformaldehyde (Ted Pella Inc., EM Grade) in PBS with $0.1 \%$ DMSO for $1 \mathrm{hr}$, followed by three rinses in PBS. All washes were carried out for $5 \mathrm{~min}$ at room temperature, unless specified otherwise. The tissue was dehydrated by sequential washes into $100 \% \mathrm{EtOH}$, frozen indefinitely at $-20^{\circ} \mathrm{C}$, and rehydrated by sequential washes into PBS. The ovaries were treated with proteinase $\mathrm{K}$ (GIBCO BRL) for $1 \mathrm{hr}(50 \mu \mathrm{g} / \mathrm{ml}$ of proteinase $\mathrm{K}$ in 50 $\mathrm{mM}$ Tris $/ \mathrm{HCl}$ at $\mathrm{pH} 7.5,50 \mathrm{~mm}$ EDTAl, and were washed twice in PBS, $0.2 \%$ glycine, once in PBS, and twice in PBT (PBS, $0.1 \%$ Tween 20 ). Samples were postfixed in $5 \%$ paraformaldehyde in PBT for $20-30 \mathrm{~min}$ and washed five times in PBT, once in $50 \%$ $\mathrm{PBT} / 50 \%$ hybridization solution, and once in $100 \%$ hybridization solution (hybridization solution: $50 \%$ formamide, $5 \times$ SSC, $50 \mu \mathrm{g} / \mathrm{ml}$ of heparin, $0.1 \%$ Tween $20,100 \mu \mathrm{g} / \mathrm{ml}$ of tRNA, 100 $\mu \mathrm{g} / \mathrm{ml}$ of single-stranded DNA). The tissue was prehybridized for $1 \mathrm{hr}$ or more at $48^{\circ} \mathrm{C}$ in hybridization solution, then hybridized with denatured probe $(3 \mu \mathrm{l}=10-20 \mathrm{ng})$ in $50 \mu \mathrm{l}$ hybridization solution overnight at $48^{\circ} \mathrm{C}-50^{\circ} \mathrm{C}$. Probe solution was removed, and the tissue was washed once for $20 \mathrm{~min}$ at $48^{\circ} \mathrm{C}$ in hybridization solution, once for $20 \mathrm{~min}$ at $48^{\circ} \mathrm{C}$ in $50 \% \mathrm{PBT} /$ $50 \%$ hybridization solution, and five times for $5 \mathrm{~min}$ at $48^{\circ} \mathrm{C}$ in PBT. Anti-digoxigenin-alkaline phosphatase antibody (Boehringer Mannheim) was diluted 1:2000 in PBT (no preabsorption), and incubated with tissue overnight at $4^{\circ} \mathrm{C}$. The tissue was washed three times for $5 \mathrm{~min}$ in PBT, three times for $20 \mathrm{~min}$ in $\mathrm{PBT}$, and twice in buffer $3(100 \mathrm{mM} \mathrm{NaCl}, 50 \mathrm{mM} \mathrm{MgCl}, 100$ $\mathrm{mm}$ Tris $/ \mathrm{HCl}$ at $\mathrm{pH}$ 9.5). Color reactions were developed in buffer 3, using $4.5 \mu \mathrm{l}$ of NBT and $3.5 \mu \mathrm{l}$ of X-phosphate per milliliter of buffer. Samples were transferred directly from the color reaction solutions into 65\% glycerol in PBS and dissected for microscopy.

Digoxigenin-labeled DNA probes were synthesized according to the manufacturer's specifications (Boehringer Mannheim), except that hexamer primers $\left[\mathrm{pd}(\mathrm{N})_{6}\right.$, Pharmacia] were added to a final concentration of $9 \mathrm{OD} / \mathrm{ml}$ to generate probes of shorter length. Labeling was carried out with 50-100 ng of gel-purified template DNA, and $1 / 10(10-20 \mathrm{ng})$ of the generated probe was used per sample.

\section{Microtubule visualization}

A protocol for microtubule fixation and staining was modified from Theurkauf et al. (1992) and Lane and Kalderon (1994). Females (2-5 days old) were dissected at room temperature in modified Robb's medium (Theurkauf et al. 1992) and fixed in $-20^{\circ} \mathrm{C}$ methanol for $10 \mathrm{~min}$. The tissue was rinsed three times in $1 \times$ PBS and extracted for $2 \mathrm{hr}$ with $1 \%$ saponin in PBS. The ovaries were rinsed twice in $0.05 \%$ saponin in PBS (PBSS) and incubated for $48 \mathrm{hr}$ at $4^{\circ} \mathrm{C}$ in a $1: 500$ dilution of monoclonal anti- $\alpha$-Tubulin antibody (Sigma, clone DM 1A) in PBSS. The samples were rinsed four times for $15 \mathrm{~min}$ each in PBSS at room temperature and incubated with a 1:100 dilution of BODIPYconjugated goat anti-mouse secondary antibody (Molecular Probes) in PBSS overnight at $4^{\circ} \mathrm{C}$ or for $2 \mathrm{hr}$ at room temperature. The samples were subsequently rinsed four times for 15 min each in PBSS, and dehydrated and mounted as described in Theurkauf et al. (1992).

Staining was observed on a Bio-Rad MRC-600 confocal microscope, utilizing the Bio-Rad COMOS program. Images were processed with NIH Image.

\section{Molecular analysis}

DNA flanking the P element was isolated from a $\lambda$ library as follows: Total genomic DNA from $h l s^{3987}$ adults was cut to completion with EcoRI, ligated into $\lambda$ arms (Stratagene), and packaged using the Gigapack Gold commercial extract from Stratagene. The library was screened with sequences from the $5^{\prime}$ end of the transposon, and 13 positive plaques were obtained. From one such phage, a $2.1-\mathrm{kb}$ EcoRI fragment that contained $0.55 \mathrm{~kb}$ of 5 ' P-element sequences, and $1.55 \mathrm{~kb}$ of genomic DNA flanking the $\mathrm{P}$ element was sublconed into Bluescript $\mathrm{KS} /+1$ (Stratagene). The flanking DNA was digested with $\mathrm{RsaI}$ and $\mathrm{ClaI}$ to obtain a fragment specific for the genomic DNA. This 0.9-kb RsaI-ClaI fragment was then used to probe two cosmid libraries Igenerous gifts of Max Scott (Massey University, Palmerston North, New Zealand) and Mark Champe]. The cosPneo Mark Champe library yielded two positive cosmids, $\mathrm{MCl}$ and $\mathrm{MC}$.

Northern analysis using a $6.5-\mathrm{kb}$ BglII cosmid fragment encompassing the P-element insertion site revealed a putative hls transcript of $4.6 \mathrm{~kb}$. A nearly full-length cDNA was isolated from an ovarian $\lambda g t 22 A$ library (Stroumbakis et al. 1994; generous gift of Peter Tolias, Public Health Research Institute, NY), using this same BglII fragment as a probe. Using sites within the polylinker (SalI, 5' end; NotI, 3' end), the 4.6-kb cDNA was subcloned into Bluescript $\mathrm{KS}|+|$, resulting in the construct pcD5.

Exonuclease III was used to construct a $5^{\prime}$ and a $3^{\prime}$ deletion series from pcD5 in a modification of the protocol described by Sambrook et al. (1989). Time-point samples were removed from exonuclease III treatment and added to tubes containing mung bean nuclease I buffer $(30 \mathrm{~mm} \mathrm{Na-acetate}$ at $\mathrm{pH} 4.6,50 \mathrm{~mm}$ $\mathrm{NaCl}, 1 \mathrm{~mm} \mathrm{Zn}$ (acetate) ${ }_{2}, 0.001 \%$ Triton X-100), and heat killed 
at $70^{\circ} \mathrm{C}$ for $15 \mathrm{~min}$. Mung bean nuclease I / 130 units; Boehringer Mannheim) then was added to each sample and incubated at $30^{\circ} \mathrm{C}$ for $30 \mathrm{~min}$, followed by addition of a stop mixture $10.3 \mathrm{M}$ Tris, $50 \mathrm{~mm}$ EDTA at $\mathrm{pH} 8.0$ ). The ends of the DNA were repaired by Klenow treatment as described by Sambrook et al. (1989), and samples were phenol-extracted in the presence of 40 $\mathrm{mM}$ Tris at $\mathrm{pH} 9.5,640 \mathrm{mM} \mathrm{LiCl}$, and $0.32 \%$ SDS. Samples then were precipitated and resuspended in ligation buffer.

Sequencing was carried out by the dideoxy chain termination method using Sequenase, version 2.0 (U.S. Biochemical), following the manufacturer's specifications. Sequence was compiled using the IntelliGenetics program. Data base searches with protein sequences were carried out using the BLAST program from the National Institutes of Health (NIH) (Altschul et al. 1990), and alignments were generated with the GCG program (University of Wisconsin, Madison).

\section{Nucleic acid hybridization}

Total RNA was prepared by the hot phenol method (Jowett 1986). RNA (35 $\mu \mathrm{g}$ ) was loaded in each lane and transferred to Zeta-Probe membrane (Bio-Rad). Hybridization at $42^{\circ} \mathrm{C}$ was according to Sambrook et al. (1989), with the addition of 0.225 $\mathrm{mg} / \mathrm{ml}$ of single-stranded DNA and $0.1 \mathrm{mg} / \mathrm{ml}$ of tRNA in the hybridization buffer.

\section{Acknowledgments}

We are grateful to Drs. Paul Schedl, Trudi Schüpbach, Bob Cohen, Lynn Cooley, and Ruth Steward for providing cDNAs, Daniel St. Johnston, Hannele Ruohola-Baker, and Jim Kennison for providing stocks, and Peter Tolias, Mark Champe, and Max Scott for providing libraries. We acknowledge the work of Todd Enderlin for help in sequencing regions of hls genomic DNA and Jennifer Sledge for aid in analyzing the $h l s^{E 616}$ and $h l s^{E 653}$ mutants. Special thanks go to Drs. Susan Parkhurst and Bob Braun and members of the Berg laboratory for critical review of the manuscript and to Drs. Heidi Horowitz and Deanna Frost for technical advice. This work was supported by NIH grants RO1 GM45248 and T32 GM07735.

The publication costs of this article were defrayed in part by payment of page charges. This article must therefore be hereby marked "advertisement" in accordance with 18 USC section 1734 solely to indicate this fact.

\section{References}

Altschul, S.F., W. Gish, W. Miller, E. Myers, and D. Lipman. 1990. Basic local alignment search tool. I. Mol. Biol. 215: 403-410.

Anderson, K. 1995. One signal, two body axes. Science 269: 489-490.

Berleth, T., M. Burri, G. Thoma, D. Bopp, S. Richstein, G. Frigerio, M. Noll, and C. Nüsslein-Volhard. 1988. The role of localization of bicoid RNA in organizing the anterior pattern of the Drosophila embryo. EMBO I. 7: 1749-1756.

Belote, J.M. and J.C. Lucchesi. 1980. Male-specific lethal mutations of Drosophila melanogaster. Genetics 96: 165-186.

Burgess, S., J.R. Couto, and C. Guthrie. 1990. A putative ATP binding protein influences the fidelity of branchpoint recognition in yeast splicing. Cell 60: 705-717.

Chasan, R. and K.V. Anderson. 1993. Maternal control of dorsal/ventral polarity and pattern in the embryo. In The development of Drosophila melanogaster. (ed. M. Bate and A. Martinez-Arias), pp. 387-424. Cold Spring Harbor Laboratory Press, Cold Spring Harbor, New York.

Chen, J.-H. and R.-J. Lin. 1990. The yeast PRP2 protein, a putative RNA-dependent ATPase, shares extensive sequence ho- mology with two other pre-mRNA splicing factors. Nucleic Acids Res. 18: 6447.

Cheung, H.-K., T.L. Serano, and R.S. Cohen. 1992. Evidence for a highly selective RNA transport system and its role in establishing the dorsoventral axis of the Drosophila egg. Development 114: 653-661.

Christerson, L.B. and D.M. McKearin. 1994. orb is required for anteroposterior and dorsoventral patterning during Drosophila oogenesis. Genes \& Dev. 8: 614-628.

Clark, I., E. Giniger, H. Ruohola-Baker, L.Y. Jan, and Y.N. Jan. 1994. Transient posterior localization of a kinesin fusion protein reflects anteroposterior polarity of the Drosophila oocyte. Curr. Biol. 4: 289-300.

Ding, D., S.M. Parkhurst, S.R. Halsell, and H.D. Lipshitz. 1993a. Dynamic Hsp83 RNA localization during Drosophila oogenesis and embryogenesis. Mol. Cell. Biol. 13: 3773-3781.

Ding, D., S.M. Parkhurst, and H.D Lipshitz. 1993b. Different genetic requirements for anterior RNA localization revealed by the distribution of ADDUCIN-like transcripts during Drosophila oogenesis. Proc. Natl. Acad. Sci. 90: 2512-2516.

Ephrussi, A., L.K. Dickinson, and R. Lehmann. 1991. oskar organizes the germ plasm and directs localization of the posterior determinant nanos. Cell 66: 37-50.

Ephrussi, B. and G.W. Beadle. 1936. A technique of transplantation for Drosophila. Am. Nat. 70: 218-225.

González-Reyes, A. and D. St. Johnston. 1994. Role of oocyte position in establishment of anterior-posterior polarity in Drosophila. Science 266: 639-642.

González-Reyes, A., H. Elliott, and D. St. Johnston. 1995. Polarization of both major body axes in Drosophila by gurkentorpedo signalling. Nature 375: 654-658.

Gutzeit, H. and R. Koppa. 1982. Time-lapse film analysis of cytoplasmic streaming during late oogenesis of Drosophila. I. Embryol. Exp. Morphol. 67: 101-111.

Hodgman, T.C. 1988. A new superfamily of replicative proteins [letter]. [published erratum appears in Nature 1988. 333: 578] Nature 333: 22-23.

Jowett, T. 1986. Preparation of nucleic acids. In Drosophila: $A$ practical approach (ed. D.B. Roberts), pp. 275-286. IRL Press, Oxford, UK.

Karpen, G.H. and A.C. Spradling. 1992. Analysis of subtelomeric heterochromatin in the Drosophila minichromosome Dp1 187 by single $P$ element insertional mutagenesis. Genetics 132: 737-753.

Kim, S.H. and R.-J. Lin. 1993. Pre-mRNA splicing within an assembled yeast spliceosome requires an RNA-dependent ATPase and ATP hydrolysis. Proc. Natl. Acad. Sci. 90: 888892.

Kim, S.-H., J. Smith, A. Claude, and R.-J. Lin. 1992. The purified yeast pre-mRNA splicing factor PRP2 is an RNA-dependent NTPase. EMBO I. 11: 2319-2326.

Kim-Ha, J., J.L. Smith, and P.M. Macdonald. 1991. oskar mRNA is localized to the posterior pole of the Drosophila oocyte. Cell 66: 23-35.

Kloc, M. and L.D. Etkin. 1995. Delocalization of Vg1 mRNA from the vegetal cortex in Xenopus oocytes after destruction of XIsirt RNA. Science 265: 1101-1103.

Koch, E.A. and R.H. Spitzer. 1983. Multiple effects of colchicine on oogenesis in Drosophila: Induced sterility and switch of potential oocyte to nurse-cell developmental pathway. Cell Tissue Res. 228: 21-32.

Kuroda, M.I., M.J. Kernan, R. Kreber, B. Ganetzky, and B.S. Baker. 1991. The maleless protein associates with the $\mathbf{X}$ chromosome to regulate dosage compensation in Drosophila. Cell 66: 935-947.

Lane, M.E. and D. Kalderon. 1994. RNA localization along the 
anteroposterior axis of the Drosophila oocyte requires PKAmediated signal transduction to direct normal microtubule organization. Genes \& Dev. 8: 2986-2995.

Lantz, V., L. Ambrosio, and P. Schedl. 1992. The Drosophila orb gene is predicted to encode sex-specific germline RNA-binding proteins and has localized transcripts in ovaries and early embryos. Development 115: 75-88.

Lasko, P. and M. Ashburner. 1988. The product of the Drosophila gene vasa is very similar to eukaryotic initiation factor-4A. Nature 335: 611-617.

Lee, C.-G. and J. Hurwitz. 1993. Human RNA helicase A is homologous to the maleless protein of Drosophila. J. Biol. Chem. 268: 16822-16830.

Lehmann, R. and C. Nüsslein-Volhard. 1991. The maternal gene nanos has a central role in posterior pattern formation of the Drosophila embryo. Development 112: 679-691.

Lin., H., L. Yue, and A. Spradling. 1994. The Drosophila fusome, a germline-specific organelle, contains membrane skeletal proteins and functions in cyst formation. Development 120: $947-956$.

Lindsley, D.L. and G.G. Zimm. 1992. The genome of Drosophila melanogaster. Academic Press, San Diego, CA.

Litman, P., J. Barg, and I. Ginzburg. 1994. Microtubules are involved in the localization of tau mRNA in primary neuronal cell cultures. Neuron 13: 1463-1474.

Lohs-Schardin, M. 1982. Dicephalic, a Drosophila mutant affecting polarity in follicle organization and embryonic patterning. Wilhelm Roux's Arch. Dev. Biol. 191: 28-36.

Mahowald, A.P. 1962. Fine structure of pole cells and polar granules in Drosophila melanogaster. J. Exp. Zool. 151: 201205.

Neuman-Silberberg, F.S. and T. Schüpbach. 1993. The Drosophila dorsoventral patterning gene gurken produces a dorsally localized RNA and encodes a TGF $\alpha$-like protein. Cell 75: $165-174$.

Peifer M., S. Orsulic, L.M. Pai, and J. Loureir. 1993. A model system for cell adhesion and signal transduction in Drosophila. Development (Suppl.) 1: 163-176.

Pokrywka, N.J. and E.C. Stephenson. 1991. Microtubules mediate the localization of bicoid RNA during Drosophila oogenesis. Development 113: 55-66.

1995. Microtubules are a general component of mRNA localization systems in Drosophila oocytes. Dev. Biol. 167: 363-370.

Ran, B., R. Bopp, and B. Suter. 1994. Null alleles reveal novel requirements for Bic-D during Drosophila oogenesis and zygotic development. Development 120: 1233-1242.

Robertson, H.M., C.R. Preston, R.W. Phillis, D.M. JohnsonSchlitz, W.K. Benz, and W.R. Engels. 1988. A stable genomic source of $P$ element transposase in Drosophila melanogaster. Genetics 118: 461-470.

Roth, S., F.S. Neuman-Silberberg, G. Barcelo, and T. Schüpbach. 1995. cornichon and the EGF receptor signaling process are necessary for both anterior-posterior and dorsal-ventral pattern formation in Drosophila. Cell 81: 967-978.

Ruohola, H., K.A. Bermer, D. Baker, F.R. Swedlow, L.Y. Jan, and Y.N. Jan. 1991. Role of neurogenic genes in establishment of follicle cell fate and oocyte polarity during oogenesis in Drosophila. Cell 66: 433-449.

Sambrook, J., E.F. Fritsch, and T. Maniatis. 1989. Molecular cloning: A laboratory manual, 2nd ed. Cold Spring Harbor Laboratory Press, Cold Spring Harbor, New York.

Schüpbach, T. and S. Roth. 1994. Dorsoventral patterning in Drosophila oogenesis. Curr. Opin. Genet. Dev. 4: 502-507.

Schüpbach, T. and E. Wieschaus. 1991. Female sterile mutations on the second chromosome of Drosophila melano- gaster. II. Mutations blocking oogenesis or altering egg morphology. Genetics 129: 1119-1136.

Schwer, B. and C. Guthrie. 1991. PRP16 is an RNA-dependent ATPase that interacts transiently with the spliceosome. $\mathrm{Na}$ ture 349: 494-499.

Shuman, S. 1992. Vaccinia virus RNA helicase: An essential enzyme related to the DE-H family of RNA-dependent NTPases. Proc. Natl. Acad. Sci. 89: 10935-10939.

Spradling, A.C. 1993. Developmental genetics of oogenesis. In The development of Drosophila melanogaster (ed. M. Bate and A. Martinez-Arias), pp. 1-70. Cold Spring Harbor Laboratory Press, Cold Spring Harbor, New York.

Stephenson, E.C. and N.J. Pokrywka. 1992. Localization of bicoid message during Drosophila oogenesis. Curr. Top. Dev. Biol. 26: 23-34.

Steward, R. and C. Nüsslein-Volhard. 1986. The genetics of the dorsal-Bicaudal-D region of Drosophila melanogaster. Genetics 113: 665-678.

St. Johnston, D. 1993. Pole plasm and the posterior group genes. In The development of Drosophila melanogaster (ed. M. Bate and A. Martinez-Arias), pp. 325-363. Cold Spring Harbor Laboratory Press, Cold Spring Harbor, New York.

St. Johnston, D., W. Driever, T. Berleth, S. Richstein, and C. Nüsslein-Volhard. 1989. Multiple steps in the localization of bicoid RNA to the anterior pole of the Drosophila oocyte. Development (Suppl.) 1: 13-19.

St. Johnston, D., D. Beuchle, and C. Nüsslein-Volhard. 1991. staufen, a gene required to localize maternal RNAs in the Drosophila egg. Cell 66: 51-63.

Stroumbakis, N.D., Z. Li, and P.P. Tolias. 1994. RNA- and single-stranded DNA-binding (SSB) proteins expressed during Drosophila melanogaster oogenesis: A homolog of bacterial and eukaryotic mitochondrial SSBs. Gene 143: 171-177.

Suter, B., L.M. Romber, and R. Steward. 1989. Bicaudal-D, a Drosophila gene involved in developmental asymmetry: Localized transcript accumulation in ovaries and sequence similarity to myosin heavy chain tail domains. Genes \& Dev. 3: $1957-1968$.

Tearle, R. and C. Nüsslein-Volhard. 1987. Tübingen mutants and stocklist. Dros. Inf. Serv. 66: 209-269.

Theurkauf, W.E. 1994a. Microtubules and cytoplasm organization during Drosophila oogenesis. Dev. Biol. 165: 352-360.

- 1994b. Premature microtubule-dependent cytoplasmic streaming in cappuccino and spire mutant oocytes. Science 265: 2093-2096.

Theurkauf, W.E., S. Smiley, M.L. Wong, and B.M. Alberts. 1992. Reorganization of the cytoskeleton during Drosophila oogenesis: Implications for axis specification and intercellular transport. Development 115: 923-936.

Theurkauf, W.E., B. Alberts, Y.N. Jan, and T. Jongens. 1993. A central role for microtubules in the differentiation of Drosophila oocytes. Development 118: 1169-1180.

Wang, C. and R. Lehmann. 1991. nanos is the localized posterior determinant in Drosophila. Cell 66: 637-647.

Wieschaus, E., J.L. Marsh, and W. Gehring. 1978. fs(1)K10, a germline-dependent female sterile mutation causing abnormal chorion morphology in Drosophila melanogaster. Wilhelm Roux's Arch. Dev. Biol. 184: 75-82.

Yisraeli, J.K., S. Sokol, and D.A. Melton. 1990. A two-step model for the localization of maternal mRNA in Xenopus oocytes: Involvement of microtubules and microfilaments in the translocation and anchoring of $\mathrm{Vgl} \mathrm{mRNA.} \mathrm{Develop-}$ ment 108: 289-298.

Yue, L. and A. Spradling. 1992. hu-li tai shao, a gene required for ring canal formation during Drosophila oogenesis, encodes a homolog of adducin. Genes \& Dev. 6: 2443-2454. 


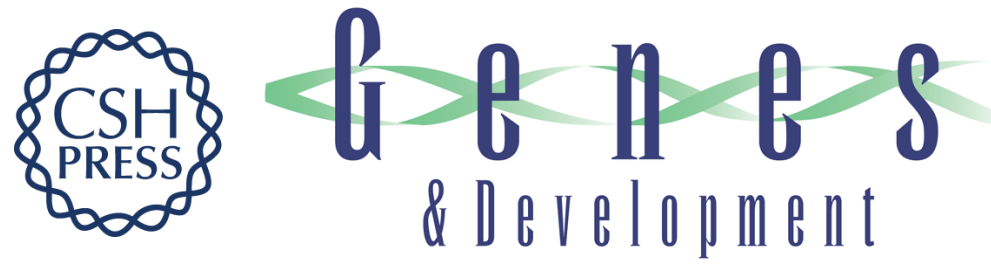

\section{Homeless is required for RNA localization in Drosophila oogenesis and encodes a new member of the DE-H family of RNA-dependent ATPases.}

D E Gillespie and C A Berg

Genes Dev. 1995, 9:

Access the most recent version at doi:10.1101/gad.9.20.2495

References This article cites 60 articles, 26 of which can be accessed free at:

http://genesdev.cshlp.org/content/9/20/2495.full.html\#ref-list-1

License

Email Alerting

Service

Receive free email alerts when new articles cite this article - sign up in the box at the top right corner of the article or click here.

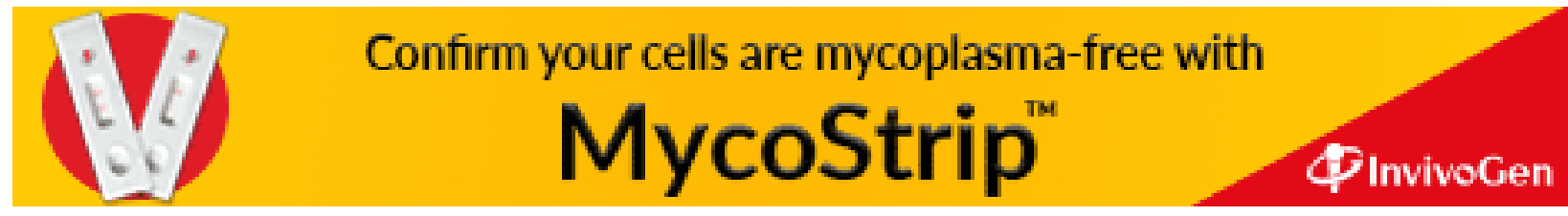

